2 Research Square
Preprints are preliminary reports that have not undergone peer review.

They should not be considered conclusive, used to inform clinical practice, or referenced by the media as validated information.

\title{
Clinical Predictors of Definitive Treatment Interruptions of Long-course Neoadjuvant Chemoradiotherapy in Locally Advanced Rectal Cancer
}

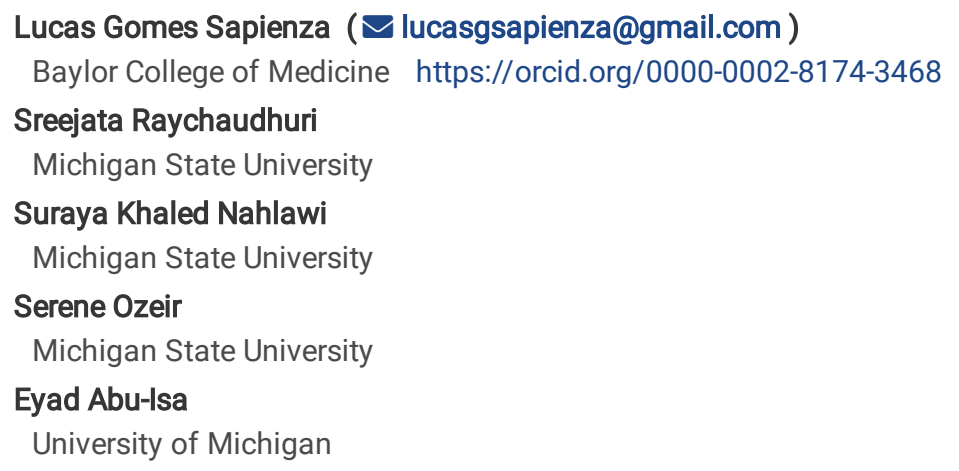

\section{Research}

Keywords: rectal cancer, radiotherapy, neoadjuvant, treatment interruption, acute toxicity

Posted Date: May 17th, 2021

DOI: https://doi.org/10.21203/rs.3.rs-489752/v1

License: (c) (i) This work is licensed under a Creative Commons Attribution 4.0 International License. Read Full License 


\section{Abstract}

Background: This study aims to identify predictors of definitive treatment interruptions (DTI) of the neoadjuvant long-course radiotherapy (LCRT) in locally advanced rectal cancer (LARC), as well as determine their impact on clinical outcomes.

Methods: Patients with stage II-III LARC treated between 2009-2018 were retrospectively analyzed $(n=101)$. Logistic regression models were performed to evaluate the impact of relevant clinical variables on grade 3 or greater (G3+) acute toxicity, definitive treatment interruption (DTI), pathological complete response (pCR), and definitive ostomy (dOST) rates. The secondary outcomes were loco-regional control (LRC), metastasis-free survival (MFS), progression-free survival (PFS), cancer-specific survival (CSS) and overall survival (OS).

Results: The incidences of grade 3 and 4 toxicities were $25.3 \%$, and $1.1 \%$, respectively. Most common G3+ toxicity were peri-anal dermatitis $(14.7 \%)$ and diarrhea $(7.4 \%)$, which were more frequent in females $(p=0.040)$ and patients with tumor closer to the anal verge $(p=0.019)$. Eleven cases $(10.9 \%)$ developed DTI, which were associated with these G3+ events $(p<0.001)$. Resection occurred after a median 7.1 weeks (IQR: 6.18.9). Downstaging occurred in $57.4 \%$ (including $17.8 \% \mathrm{pCR}$ ), $88 \%$ achieved negative margins and the dOST rate was $56.4 \%$. After 49.5 months median follow-up, the 5-year LRC, MFS, PFS, CSS and OS were: $94.4 \%, 78.9 \%, 74.7 \%, 85.2 \%$ and $81.6 \%$, respectively. DTI events did not impact $\operatorname{pCR}(p=0.435)$, dOST $(p=0.611)$ or any survival outcomes. The only factors associated with loco-regional failure were close/positive margins $(p<0.001)$ and pathologic stage yplII $(p=0.002)$.

Conclusions: Tumors close to the anal verge and female sex were associated with increased G3+ toxicity, which was predictive of DTI. The resultant partial or complete omission of the planned boost, however, dose did not increase the chance of local recurrence. Further studies to clarify the benefit and the best timing to deliver the boost are warranted, especially for the patients with positive margins.

\section{Introduction}

Locally advanced rectal cancer (LARC) is better controlled with the combination of two local treatments. Influenced by the results of the CAO/ARO/AIO-94 [1, 2] and NSABP R-03 studies [3], the current standard of care favors initiating with radiotherapy (RT) followed by surgical resection. The most used neoadjuvant RT regimens are long course (LCRT), which delivers 25-28 fractions (1.8-2.0 Gy) with concurrent chemotherapy; and short course (SCRT), consisting of 25 Gy in 5 fractions of $5.0 \mathrm{~Gy}$.

The relatively higher prescription dose utilized in the LCRT requires a more protracted delivery (5-6 weeks vs. 1 week for SCRT). The possible increased tumor downstaging rate [4-6] comes at the expense of more acute toxicity events. These on-treatment toxicity events can reduce treatment compliance causing definitive treatment interruptions (DTI). To illustrate that, the neoadjuvant arm of the CAO/ARO/AIO-94 trial [1] reported that $27 \%$ of patients developed G3 + toxicity and $8 \%$ did not receive total radiotherapy dose. In the same direction, $8.0 \%, 7.4 \%$ and $3.0 \%$ of the patients did not receive full RT dose in the LCRT (with fluorouracil) arms of STAR-01 [7], TROG 01.04 [8], and CAO/ARO/AIO-04 [9] studies, respectively. To date, it is unclear whether these DTIs affect prognosis in LARC.

A detailed examination of the factors causing these DTI could provide relevant insights to improve the RT technique, including indirect evidence regarding the optimal prescription dose. Based on that, we reviewed our experience with the main objectives: I) calculate the rate of cases that developed definitive treatment interruption (DTI); II) analyze factors that predispose to DTI; and III) evaluate the impact of DTI on outcomes.

\section{Materials And Methods}

All patients with diagnosis of rectal cancer referred to the radiation oncology department between 2009 and 2018 were retrospectively screened $(n=211)$. After applying the exclusion criteria (Supplementary Figure S1), we included 101 consecutive patients with LARC (American Joint Committee on Cancer - AJCC stages II and III) that received long course neoadjuvant chemoradiotherapy followed by surgical resection. The institutional review board (IRB) approved this study design and the use of patient information without individual identification (IRB number 1466256-1).

\subsection{Treatment Protocol}

Radiotherapy consisted of $45 \mathrm{~Gy}$ ( 25 daily fractions of $1.8 \mathrm{~Gy}$ ) to the pelvis plus $5.4 \mathrm{~Gy}$ (additional 3 fractions of $1.8 \mathrm{~Gy}$ ) to the gross disease, including primary tumor and suspicious enlarged regional nodes. The mesorectum, presacral nodes, and internal iliac nodes were electively covered for all cases. Cases with extension to anterior pelvic structures (prostate, uterus, or bladder) had the external iliac chain prophylactically treated. Of the 22 cases with tumors involving or extending below the dentate line (defined as $2.1 \mathrm{~cm}$ above the anal verge [10]), the bilateral inguinal lymph nodes were electively covered in 7 patients (31.8\%), per radiation oncologist preference. Concurrent chemotherapy consisted of fluoropyrimidine-based (5-FU or capecitabine). Surgery was performed using the principles of total mesorectal excision (TME). Additional adjuvant chemotherapy was used after surgery per medical oncologist discretion.

\subsection{Endpoints}

Page $2 / 12$ 
The rates of grade 3 or greater (G3+) acute toxicity, definitive treatment interruption (DTI), pathological complete response (pCR: ypTOypN0), and definitive ostomy (dOST: no stoma at the last follow-up) are the main study outcomes. Toxicity was obtained from the on-treatment weekly evaluations performed by the radiation oncologist. These were graded retrospectively according to the general guidelines of the Common Terminology Criteria for Adverse Events v4.0 [11]. The toxicity analysis was based on 95 instead of 101 cases as no toxicity description was found in the electronic medical records for 6 cases. The secondary study outcomes are loco-regional control (LRC), metastasis-free survival (MFS), progression-free survival (PFS; composite of locoregional failure, distant failure, or death), cancer-specific survival (CSS) and overall survival (OS). The initial time for the time-to event endpoints was defined as the date of the end radiotherapy.

\subsection{Statistical analysis}

The univariate analysis was performed using logistic regression (categorical) and log-rank test (time-dependent). For the main binary outcomes, variables with $p<0.25$ were incorporated in the multivariate logistic regression model. Due to the reduced number of events and the fact that DTI did not affect time-dependent outcomes (as shown in the Sect. 3.4 Disease control and survival), a multivariate analysis (Cox regression model) of the survival outcomes was not performed. The time dependent endpoints were analyzed via the Kaplan-Meier method [12], with patient death included as a censoring event for LRC and MFS endpoints. All statistical analyses were performed using IBM SPSS Statistics, Build 1.0.0.1508, Armonk, NY, USA. Graphic representation of the logistic regression model was performed using the Microsoft Excel, Version 16.57.1, Redmond, WA, USA.

\section{Results}

\subsection{Patients and treatment characteristics}

The median age of the cohort was 60.6 years (IQR 53.1-69.1). Most common symptoms at presentation were rectal bleeding (79.2\%), rectal pain (14.9\%) and constipation (10.9\%) [Supplementary Table S1]. Fifty-six percent were male and 17.8\% were active smokers at the time of diagnosis. Twenty-five percent of the females (11/44) had hysterectomy prior to the diagnosis of rectal cancer for benign causes. The median initial carcinoembryonic antigen (CEA) was $3.2 \mathrm{ng} / \mathrm{mL}$ (IQR: $1.9-7.5$ ) and $51.5 \%$ of patients were stage III.

The majority of patients (82.2\%) were treated with 3D conformal radiotherapy (3DCRT) and 17.8\% were treated with intensity-modulated radiation therapy (IMRT) or volumetric modulated arc therapy (VMAT). Ninety-seven percent of the cases received concurrent fluoropyrimidinebased chemotherapy. Surgery involved abdominal perineal resection (APR) in $47.5 \%$ of the cases. Minimally invasive surgery (MIS) was used in $56.4 \%$ of the patients. Additional patient and treatment characteristics were presented in Table 1 and Supplementary Table S2. 
Table 1

- Patient and treatment characteristics.

\begin{tabular}{|c|c|c|c|}
\hline Characteristic & $\begin{array}{l}\text { Overall } \\
\mathbf{N}(\%)\end{array}$ & Median (mean) & IQR (25-75\%) \\
\hline Age (years) & 101 & $60.6(60.8)$ & $53.1-69.1$ \\
\hline \multicolumn{4}{|l|}{ Sex } \\
\hline Male & $57(56.4)$ & - & - \\
\hline Female & $44(43.6)$ & - & - \\
\hline BMI $\left(\mathrm{kg} / \mathrm{m}^{2}\right)$ & 99 & $27.7(28.5)$ & $24.4-31.2$ \\
\hline \multicolumn{4}{|l|}{ Smoking } \\
\hline Non-current (never or former) & $83(82.2)$ & - & - \\
\hline Current & $18(17.8)$ & - & - \\
\hline Initial CEA (ng/mL) & 92 & $3.2(8.1)$ & $1.9-7.5$ \\
\hline Distance from AV (cm) & 100 & $6.0(6.2)$ & $3.0-9.0$ \\
\hline Initial Tumor Size (cm) & 86 & $4.8(4.9)$ & $3.0-6.0$ \\
\hline \multicolumn{4}{|l|}{ Clinical AJCC stage } \\
\hline ॥ & $48(47.5)$ & - & - \\
\hline III & $52(51.5)$ & - & - \\
\hline NA & $1(1.0)$ & - & - \\
\hline \multicolumn{4}{|l|}{ RT Technique } \\
\hline 3DCRT & $83(82.2)$ & - & - \\
\hline IMRT/VMAT & $18(17.8)$ & - & - \\
\hline \multicolumn{4}{|l|}{ Energy } \\
\hline $6 \mathrm{MV}$ & $11(10.9)$ & - & - \\
\hline $15 \mathrm{MV}$ & $90(89.1)$ & - & - \\
\hline \multicolumn{4}{|l|}{ RT timing } \\
\hline AM & $67(66.3)$ & - & - \\
\hline PM & $34(22.7)$ & - & - \\
\hline Time from RT to surgery (weeks) & 101 & $7.1(7.6)$ & $6.1-8.9$ \\
\hline \multicolumn{4}{|l|}{ Type of Surgery } \\
\hline open & $40(39.6)$ & - & - \\
\hline MIS - laparoscopic or robotic & $57(56.4)$ & - & - \\
\hline NA & $4(4.0)$ & - & - \\
\hline \multicolumn{4}{|l|}{ Surgical Procedure } \\
\hline LAR & $53(52.5)$ & - & - \\
\hline APR & $48(47.5)$ & - & - \\
\hline \multicolumn{4}{|l|}{ Margins } \\
\hline negative & $89(88.1)$ & - & - \\
\hline
\end{tabular}

IQR: interquartile range. BMI: body mass index. CEA: carcinoembryonic antigen. AV: anal verge. AJCC American Joint Committee on Cancer. pCR: pathological complete response. RT: radiotherapy. 3DCRT: 3D-conformal radiotherapy. IMRT: intensity-modulated radiation therapy. VMAT: volumetric modulated arc therapy. MV: megavolt. MIS: minimally invasive surgery. LAR: low anterior resection. APR: abdominal perineal resection. NA: not available. 


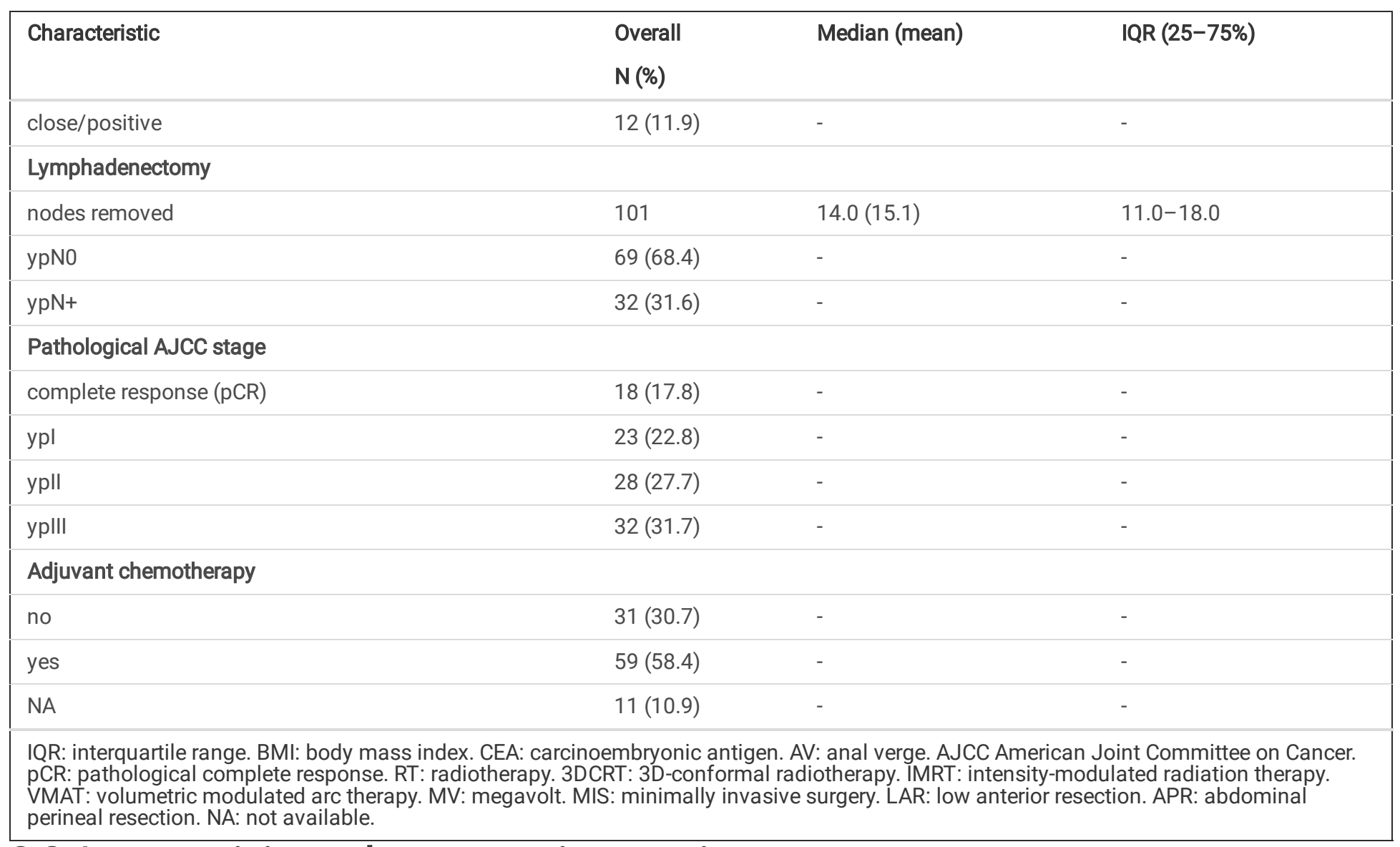

\subsection{Acute toxicity and treatment interruptions}

The incidences of grade $0,1,2,3$ and 4 toxicities were: $2.1 \%, 41.0 \%, 30.5 \%, 25.3 \%$, and $1.1 \%$, respectively. Most common G3 + toxicity were perianal dermatitis $(14.7 \%)$ and diarrhea $(7.4 \%)$. The G3 + events were more frequent in females (OR 2.84, $p=0.040)$ and patients with tumor close to the anal verge (AV) (continuous, each centimeter from AV to proximal rectum: $O R=.85, p=0.019$, Fig. 1). A detailed description of the acute toxicity events is provided in table format in the Supplementary Tables S3 and S4.

Eleven cases (10.9\%) developed DTI which occurred before or at fraction number 25 in five patients (45\%) and during the boost phase (before fractions 26,27 or 28 ) in 6 cases (55\%). One patient had a bowel perforation after fraction number 17 of the pelvic field, requiring immediate surgery.

Table 2 describes the grade $2+$ events that each patient that developed DTI experienced during RT. In this subgroup, the most frequent G3 + toxicities were diarrhea (45.5\%), peri-anal dermatitis (18.2\%) and weight loss $(18.2 \%)$. The only variable associated with DTI was G3 + toxicity (OR 50.00, $p<0.001$, Table 3 and Supplementary Tables S5-6). Among female patients, previous hysterectomy was not associated with grade 3 + toxicity $(12 / 32$ vs. $3 / 11, p=0.541)$. 
Table 2

) Cases with DTI and related toxicity.

\begin{tabular}{|c|c|c|c|c|c|c|c|c|c|c|c|}
\hline Case & $\begin{array}{l}\text { RT Fractions } \\
\text { (delivered/total) }\end{array}$ & $\begin{array}{l}\text { Maximal } \\
\text { Toxicity }\end{array}$ & Diarrhea & $\begin{array}{l}\text { Peri-Anal } \\
\text { Dermatitis }\end{array}$ & $\begin{array}{l}\text { Weight } \\
\text { Loss }\end{array}$ & $\begin{array}{l}\text { Bowel } \\
\text { Perforation }\end{array}$ & $\begin{array}{l}\text { Rectal } \\
\text { Pain }\end{array}$ & Emesis & Fatigue & Nausea & Dysuria \\
\hline 1 & $17 / 28$ & 4 & 1 & 2 & 1 & 4 & 2 & 0 & 1 & 0 & 0 \\
\hline 2 & $26 / 28$ & 3 & 2 & 0 & 0 & 0 & 3 & 0 & 2 & 2 & 2 \\
\hline 3 & $25 / 28$ & 3 & 3 & 0 & 0 & 0 & 0 & 0 & 1 & 0 & 0 \\
\hline 4 & $26 / 28$ & 3 & 3 & 0 & 0 & 0 & 2 & 0 & 0 & 0 & 2 \\
\hline 5 & $27 / 28$ & 3 & 3 & 0 & 0 & 0 & 1 & 0 & 2 & 2 & 0 \\
\hline 6 & $27 / 28$ & 3 & 3 & 0 & 1 & 0 & 0 & 1 & 1 & 1 & 0 \\
\hline 7 & $23 / 28$ & 3 & 0 & 0 & 3 & 0 & 0 & 3 & 2 & 2 & 0 \\
\hline 8 & $26 / 28$ & 3 & 2 & 3 & 0 & 0 & 0 & 0 & 2 & 1 & 0 \\
\hline 9 & $25 / 28$ & 3 & 3 & 0 & 3 & 0 & 0 & 0 & 0 & 0 & 0 \\
\hline 10 & $23 / 28$ & 2 & 2 & 0 & 0 & 0 & 0 & 0 & 0 & 0 & 1 \\
\hline 11 & $27 / 28$ & 3 & 2 & 3 & 1 & 0 & 0 & 0 & 0 & 0 & 0 \\
\hline \multicolumn{3}{|c|}{ G3 + with DTI/total cases with DTI } & $\begin{array}{l}45.5 \% \\
(5 / 11)\end{array}$ & $\begin{array}{l}18.2 \% \\
(2 / 11)\end{array}$ & $\begin{array}{l}18.2 \% \\
(2 / 11)\end{array}$ & $\begin{array}{l}9.1 \% \\
(1 / 11)\end{array}$ & $\begin{array}{l}9.1 \% \\
(1 / 11)\end{array}$ & $\begin{array}{l}9.1 \% \\
(1 / 11)\end{array}$ & $0 \%$ & $0 \%$ & $0 \%$ \\
\hline \multicolumn{3}{|c|}{ G3 + with $\mathrm{DTI} /$ total cases with $\mathrm{G} 3+$} & $\begin{array}{l}71.4 \% \\
(5 / 7)\end{array}$ & $\begin{array}{l}14.3 \% \\
(2 / 14)\end{array}$ & $\begin{array}{l}100 \% \\
(2 / 2)\end{array}$ & $100 \%(1 / 1)$ & $\begin{array}{l}100 \% \\
(1 / 1)\end{array}$ & $\begin{array}{l}100 \% \\
(1 / 1)\end{array}$ & $0 \%$ & $0 \%$ & $0 \%$ \\
\hline \multicolumn{3}{|c|}{ total cases with G3+/ total cases } & $\begin{array}{l}7.2 \% \\
(7 / 95)\end{array}$ & $\begin{array}{l}14.7 \% \\
(14 / 95)\end{array}$ & $\begin{array}{l}2.1 \% \\
(2 / 95\end{array}$ & $\begin{array}{l}1.1 \% \\
(1 / 95)\end{array}$ & $\begin{array}{l}1.1 \% \\
(1 / 95)\end{array}$ & $\begin{array}{l}1.1 \% \\
(1 / 95)\end{array}$ & $0 \%$ & $0 \%$ & $0 \%$ \\
\hline
\end{tabular}

Table 3

Multivariate analysis.

\begin{tabular}{|c|c|c|c|c|}
\hline Endpoint & Variable & Category & Odds Ratio (95\% Cl) & $p$-value \\
\hline \multirow{3}{*}{$\begin{array}{l}\text { G3 + toxicity } \\
(n=94) *\end{array}$} & Sex & Male & Reference & \\
\hline & & Female & $2.841(1.050-7.692)$ & 0.040 \\
\hline & Distance to AV & Continuous & $0.847(0.737-0.974)$ & 0.019 \\
\hline \multirow{2}{*}{$\begin{array}{l}\text { DTI } \\
(n=95) \star *\end{array}$} & G3 + toxicity & No & Reference & \\
\hline & & Yes & $50.000(5.917-422.520)$ & $<0.001$ \\
\hline \multirow{4}{*}{$\begin{array}{l}\mathrm{pCR} \\
(\mathrm{n}=85) \text { * }\end{array}$} & cT size & Continuous & $0.460(0.280-0.756)$ & 0.002 \\
\hline & Distance to AV & Continuous & $1.249(1.014-1.538)$ & 0.036 \\
\hline & Time RT to surgery & $<6$ weeks & Reference & \\
\hline & & $\geq 6$ weeks & $9.144(0.851-98.310)$ & 0.068 \\
\hline \multirow[t]{3}{*}{ Definitive Ostomy } & Distance to $\mathrm{AV}$ & Continuous & $0.647(0.531-0.787)$ & $<0.001$ \\
\hline & yp AJCC Stage & ypl-II & Reference & \\
\hline & & yplll & $2.939(0.859-10.060)$ & 0.086 \\
\hline
\end{tabular}

\subsection{Pathological response and ostomy}

Surgical resection occurred after a median of 7.1 weeks (IQR: 6.1-8.9) from the end of neoadjuvant therapy and consisted of total meso-rectal excision. Relatively to the initial clinical AJCC group stage, $57.4 \%$ had downstaging (including $17.8 \% \mathrm{pCR}$ ), $25.7 \%$ had no change in stage, and 
$15.8 \%$ had more advanced disease in the final pathology report. Eighty-eight percent achieved negative margins. The dOST rate was $56.4 \%$ (57/101). DTI events did not impact pCR rate (OR 0.43, $p=0.435)$ or dOST (OR 1.40, $p=0.611)$ (Table 3 and Supplementary Tables S7-S8).

\subsection{Disease control and survival}

After 49.5 months median (mean 56.7 months) follow-up interval, three patients developed loco-regional failure (3y/5y LRC 97.8\%/94.4\%) (Fig. 2 ABC). DTI event was not associated with LRC (5y $100 \%$ with DTI vs. $97.5 \%$ without DTI, $p=0.534$ ) [Figure $3 \mathrm{~A}$ ]. The only factors associated with local failure were close/positive margins (5y LRC 76.2\% vs. 100\% for negative margins, $p<0.001$ ) and pathologic stage ypllI (5y LRC $92.3 \%$ vs. $100 \%$ for $\mathrm{pCR} /$ stage ypl/stage ypll, $p=0.002$ ) [Supplementary Table S9].

Nineteen patients developed distant metastasis, which more commonly involved non-regional lymph nodes (12/19=63.1\%); lung (10/19= $52.6 \%)$, and liver $(9 / 19=47.4 \%)$ (Supplementary Table S10). The 3y/5y MFS, PFS, CSS and OS were: $85.8 \% / 78.9 \%, 83.0 \% / 74.7 \%, 90.3 \% / 85.2 \%$ and $88.4 \% / 81.6 \%$, respectively. DTI events were not associated with MFS $(p=0.946)$, PFS $(p=0.509)$, CSS $(p=0.584)$ or OS $(p=0.974)$ [Figure 3 $\mathrm{BC}$.

\section{Discussion}

To our knowledge, the present study is the first to focus on the events of definitive interruption of RT delivery in the neoadjuvant treatment of LARC. We found that one in every ten patients treated with long course chemoradiotherapy presented DTI and that these events were strongly associated with G3 + toxicity. Although these unexpected interruptions did not impact oncologic outcomes (including pathological response, need of definitive ostomy and local control), they indicate that improvements in treatment tolerability are necessary.

Grade 3 diarrhea can be defined as 7 or more stools per day over baseline limiting self-care activities of daily living [11]. In our cohort, these events occurred in less than $10 \%$ of the total cases, however it preceded almost $50 \%$ of the DTI events. The secondary dehydration and reduction in performance status due to this side effect plays an important role in the decision to suspend treatment. Intriguingly, female patients experienced higher rates of grade $3+$ toxicity. Similar association with sex was previously described by a group from the University of Calgary [13] which hypothesized that the anatomical changes of hysterectomized patients (4 of 11 women in their series) were responsible for such increase in toxicity by exposing more small bowel in the treatment field. In our study, however, no association between absence of uterus at the time of RT and toxicity ( $p=0.541)$ was noted. In addition to that, tumors close to the AV were associated with more toxicity indicating that a better understanding of the dose constraints for organs at risk in the lower pelvis $[13,14]$ and of the gastro-intestinal physiology $[15-16]$ have the potential to improve tolerability.

Despite having a reduction in the RT dose delivered, cases with DTI did not have worse oncologic outcomes, including pCR and local control. This observation indicates that doses higher than $45 \mathrm{~Gy}$ may not be necessary for LCRT. In that respect, researchers from the Toronto (Canada) previously compared the results of three phase II studies that used $40 \mathrm{~Gy}, 46 \mathrm{~Gy}$ and $50 \mathrm{~Gy}$ in the neoadjuvant setting [17]. They found a higher pCR rate with increasing the dose ( $15 \%$ vs. $23 \%$ vs. $33 \%$, respectively, $p=0.07$ ), but no improvement in $2 y$-local recurrence-free survival above 46 Gy $(72 \%$ vs. $90 \%$ vs. $89 \%, p=0.02)$. Of note, the high-rate of local recurrences reported may indicate that TME was not performed for all cases and possibly more utilized in the later protocols with high doses, disfavoring the lower dose arm. More recently, some attempts to further escalate the neoadjuvant RT dose with TME were performed. One phase III study from Denmark and Canada [18] compared two radiation doses (EQD2 $49.6 \mathrm{~Gy}_{10}$ vs. $62.1 \mathrm{~Gy}_{10}$ ) showing same pCR in both arms (18\% vs. 18\%). Similarly, the RECTAL-BOOST trial conducted in the Netherlands [19] failed to improve pathological complete response (for operable cases) or 2-year sustained clinical response (for watch-and-wait cases) with an escalated boost delivering an EQD2 of $66.3 \mathrm{~Gy}_{10}$ (compared to EQD2 $50 \mathrm{~Gy}_{10}$ ).

A comparison between the prescription doses of SCRT and LCRT gives another relevant perspective. Applying the normalization by the equivalent dose in $2 \mathrm{~Gy}$ fractions (EQD2) and assuming a/ $\beta=10$, the LCRT regimen of 45 Gy in 5 weeks (25 x 1.8Gy, EQD2 44.2Gy 10 ) delivers $40 \%$ more dose when compared with SCRT ( $5 \times 5 \mathrm{~Gy}$, EQD2 $31.2 \mathrm{~Gy}_{10}$ ). This increase raises to $60 \%$ when using the fractionation of $50 \mathrm{~Gy}$ in five weeks ( 25 x 2Gy, EQD2 $50 \mathrm{~Gy}_{10}$ ) or adding a 5.4 Gy boost $\left(28\right.$ x $1.8 \mathrm{~Gy}$, EQD2 $49.6 \mathrm{~Gy}_{10}$ ). Despite higher doses in LCRT, the local control of both LCRT and SCRT strategies are similar [20].

Cases with close or positive resection margins after TME had significant worse loco-regional control when compared with patients with negative margins (5-year: $76.2 \%$ vs. $100 \%, p<0.001$ ). Based on that and the fact the pre-operative boost did not improve local control, it could be hypothesized that omitting the pre-operative boost in order to leave room for additional radiation dose intra- [21] or post-operatively [22] could potentially benefit the subgroup of patients that failed to achieve clear margins [23-25] or at the time of a local recurrence [26]. The postoperative indication of boost has an enticing prospect, especially if no further therapy is planned after resection, which is the case in the total neoadjuvant therapy strategy [27-29]. For patients with tumors involving the anatomical anal canal (at or below dentate line) [10], the risk/benefit ratio is even more unfavorable regarding the pre-operative boost. These patients have a higher risk of grade $3+$ toxicity and no benefit of sphincter preservation since they require abdominal perineal resection and colostomy, independently of the theoretical additional downstaging effect of the boost. Importantly, tailoring the indication of boost based on pathological findings could spare unnecessary boost for

Page $7 / 12$ 
patients with clear margins ( $88 \%$ in our cohort), automatically reducing the incidence of DTI by about half ( $55 \%$ of cases had interruption during the boost phase).

Limitations of the present study include its retrospective design and relatively modest sample size, which precluded a more robust multivariate analysis of the secondary survival outcomes. In addition, no data on chemotherapy tolerability (dose reduction events and total number of cycles delivered) and patient reported outcomes were available, which could provide more comprehensive insights regarding treatment tolerability. Importantly, the findings of the present study do not apply to the scenario of organ preservation where radiation is potentially the only local treatment.

In summary, we found that one in every ten patients with LARC treated with LCRT presented a DTI event, which was strongly related to grade $3+$ toxicity. These treatment interruptions affected the delivery of the boost before surgery, without affecting pCR, definitive ostomy rates, and local control. In addition, patients with positive margins after surgery had worse local control and survival, raising the question of whether the boost should be reserved for those that failed to achieve negative margins and delivered after surgery.

\section{Declarations}

Ethics approval and consent to participate: All procedures performed in studies involving human participants were in accordance with the ethical standards of the institutional and/or national research committee and with the 1964 Helsinki Declaration and its later amendments or comparable ethical standards. This article does not contain any studies with animals performed by any of the authors. This study was approved by the Ascension Providence Hospital Institutional Review Board (Michigan, USA) and the requirement for informed consent was waived given the retrospective nature of the data.

Consent for publication: Not applicable.

Availability of data and material: The datasets used and/or analyzed during the current study are available from the corresponding author on reasonable request.

Competing interests: The authors declare that there is no conflict of interest regarding the publication of this paper.

Funding: This research did not receive any specific grant from funding agencies in the public, commercial, or not-for-profit sectors.

Authors' contributions: LGS, SR and EA designed the study. LGS, SR, SKN, SO and EA supervised data abstraction and vouch for the data. LGS and EA analyzed the data and vouch for it. LGS, SR and EA wrote the first draft of the manuscript. All authors read and approved the final manuscript.

Acknowledgments: none.

\section{References}

1. - Sauer R, Becker H, Hohenberger W, et al. Preoperative versus postoperative chemoradiotherapy for rectal cancer. N Engl J Med. 2004;351(17):1731-40.

2. - Sauer R, Liersch T, Merkel S, et al. Preoperative versus postoperative chemoradiotherapy for locally advanced rectal cancer: results of the German CAO/ARO/AIO-94 randomized phase III trial after a median follow-up of 11 years. J Clin Oncol. 2012;30(16):1926-33.

3. - Roh MS, Colangelo LH, O'Connel MJ, et al. Preoperative multimodality therapy improves disease-free survival in patients with carcinoma of the rectum: NSABP R-03. J Clin Oncol. 2009;27(31):5124-30.

4. - Latkauskas T, Pauzas H, Gineikiene I, et al. Initial results of a randomized controlled trial comparing clinical and pathological downstaging of rectal cancer after preoperative short-course radiotherapy or long-term chemoradiotherapy, both with delayed surgery. Colorectal Dis. 2012;14(3):294-8.

5. - Hoendervangers S, Couwenberg AM, Intven MPW, van Grevenstein WMU, Verkooijen HM. Comparison of pathological complete response rates after neoadjuvant short-course radiotherapy or chemoradiation followed by delayed surgery in locally advanced rectal cancer. Eur $\mathrm{J}$ Surg Oncol. 2018;44(7):1013-7.

6. - Rombouts AJM, Hugen N, Verhoeven RHA, et al. Tumor response after long interval comparing 5x5Gy radiation therapy with chemoradiation therapy in rectal cancer patients. Eur J Surg Oncol. 2018;44(7):1018-24.

7. - Aschele C, Cionini L, Lonardi S, et al. Primary tumor response to preoperative chem-radiation with or without oxaliplatin in locally advanced rectal cancer: pathologic results of the STAR-01 randomized phase III trial. J Clin Oncol. 2011;29(20):2773-80.

8. - Ansari N, Solomon MJ, Fisher RJ, et al. Acute adverse events and postoperative complications in a randomized trial of preoperative shortcourse radiotherapy versus long-course chemoradiotherapy for T3 adenocarcinoma of the rectum: Trans-Tasman Radiation Oncology Group

Page 8/12 
Trial (TROG 01.04). Ann Surg. 2017;265(5):882-8.

9. - Rödel C, Liersch T, Becker H, et al. Preoperative chemoradiotherapy and postoperative chemotherapy with fluorouracil and oxaliplatin versus fluorouracil alone in locally advancer rectal cancer: initial results of the German CAO/ARO/AIO-04 randomized phase 3 trial. Lancet Oncol. 2012;13(7):679-87.

10. - Nivatvongs S, Stern HS, Fryd DS. The length of the anal canal. Dis Colon Rectum. 1981;24(8):600-1.

11. - Common terminology criteria for adverse events v4.0 (CTCAE). U.S. Department of Health and Human Services - National Institutes of Health. Available: https://www.eortc.be/services/doc/ctc/ctcae_4.03_2010-06-14_quickreference_5x7.pdf [Accessed 03 April 2021].

12. - Kaplan EL, Meier P. Nonparametric estimation from incomplete observations. J Am Stat Assoc. 1958;53:457-81.

13. - Banerjee R, Chakraborty S, Nygren I, Sinha R. Small bowel dose parameters predicting grade $\geq 3$ acute toxicity in rectal cancer patients treated with neoadjuvant chemoradiation: an independent validation study comparing peritoneal space versus small bowel loop contouring techniques. Int J Radiat Oncol Biol Phys. 2013;85(5):1225-31.

14. - Kavanagh BD, Pan CC, Dawson LA, et al. Radiation dose-volume effects in the stomach and small bowel. Int J Radiat Oncol Biol Phys. 2010;76(3 Suppl):101-7.

15. - Ferreira MR, Andreyev JH, Mohammed K, et al. Microbiota- and radiotherapy-induced gastrointestinal side-effects (MARS) study: a large pilot study of the microbiome in acute and late-radiation enteropathy. Clin Cancer Res. 2019;25(21):6487-500.

16. - Mitra A, Biegert GWG, Delgado AY, et al. Microbial diversity and composition is associated with patient-reported toxicity during chemoradiation therapy for cervical cancer. Int J Radiat Oncol Biol Phys. 2020;107(1):163-71.

17. - Wiltshire KL, Ward IG, Swallow C, et al. Preoperative radiation with concurrent chemotherapy for resectable rectal cancer: effect of dose escalation on pathologic complete response, local recurrence-free survival, disease-free survival, and overall survival. Int J Radiat Oncol Biol Phys. 2006;64(3):709-16.

18. - Jakobsen A, Ploen J, Vuong T, et al. Dose-effect relationship in chemoradiotherapy for locally advanced rectal cancer: a randomized trial comparing two radiation doses. Int J Radiat Oncol Biol Phys. 2012;84(4):949-54.

19. - Couwenberg AM, Burbach JPM, Berbee M, et al. Efficacy of dose-escalated chemoradiation on complete tumor response in patients with locally advanced rectal cancer (RECTAL-BOOST): a phase 2 randomized controlled trial. Int J Radiat Oncol Biols Phys. 2020;108(4):100818.

20. - Erlandsson J, Holm T, Pettersson D, et al. Optimal fractionation of preoperative radiotherapy and timing to surgery for rectal cancer (Stockholm III): a multicentre, randomised, non-blinded, phase 3, non-inferiority trial. Lancet Oncol. 2017;18(3):336-46.

21. - Mirnezami R, Chang GJ, Das P, et al. Intraoperative radiotherapy in colorectal cancer: systematic review and meta-analysis of techniques, long-term outcomes, and complications. Surg Oncol. 2013;22(1):22-35.

22. - Dagoglu N, Nedea E, Poylin V, Nagle D, Mahadevan A. Post operative stereotactic radio surgery for positive or close margins after preoperative chemoradiation and surgery for rectal cancer. J Gastrointest Oncol. 2016;7(3):315-20.

23. - de Nes LCF, Drager LD, Verstegen MG, et al. Persistent high rate of positive margins and postoperative complications after surgery for cT4 rectal cancer at a national level. Dis Colon Rectum. 2021;64(4):389-98.

24. - Sung S, Kim SH, Lee JH, et al. Continuos effect of radial resection margin on recurrence and survival in rectal cancer patients who receive preoperative chemoradiation and curative surgery: a multi center retrospective analysis. Int J Radiat Oncol Biol Phys. 2017;98(3):647-53.

25. - Zeng WG, Liu MJ, Zhou ZX, Wang ZJ. A distal resection margin of $\leq 1 \mathrm{~mm}$ and rectal cancer recurrence after sphincter-preserving surgery: the role of a positive distal margin in rectal cancer surgery. Dis Colon Rectum. 2017;60(11):1175-83.

26. - Valentini V, Morganti AG, Gambacorta MA, et al. Preoperative hyperfractionated chemoradiation for locally recurrent rectal cancer in patients previously irradiated to the pelvis: a multicentric phase II study. Int J Radiat Oncol Biol Phys. 2006;64(4):1129-39.

27. - Fernandez-Martos C, Pericay C, Aparicio J, et al. Phase II, randomized study of concomitant chemoradiotherapy followed by surgery and adjuvant capecitabine plus oxaliplatin (CAPOX) compared with induction CAPOX followed by concomitant chemoradiotherapy and surgery in magnetic resonance imaging-defined, locally advanced rectal cancer: Group cancer de recto 3 study. J Clin Oncol. 2010;28:859-65.

28. - Fernandez-Martos C, Garcia-Albeniz X, Pericay C, et al. Chemoradiation, surgery and adjuvant chemotherapy versus induction chemotherapy followed by chemoradiation and surger: long-term results of the Spanish GCR-3 phase II randomized trial. Ann Oncol. 2015;16(8):1722-8.

29. - Fokas E, Allgauer M, Polat B, et al. Randomized phase II trial of chemoradiotherapy plus induction or consolidation chemotherapy as total neoadjuvant therapy for locally advanced rectal cancer: CAO/ARO/AIO-12. J Clin Oncol. 2019;37(34):2112-22.

\section{Figures}




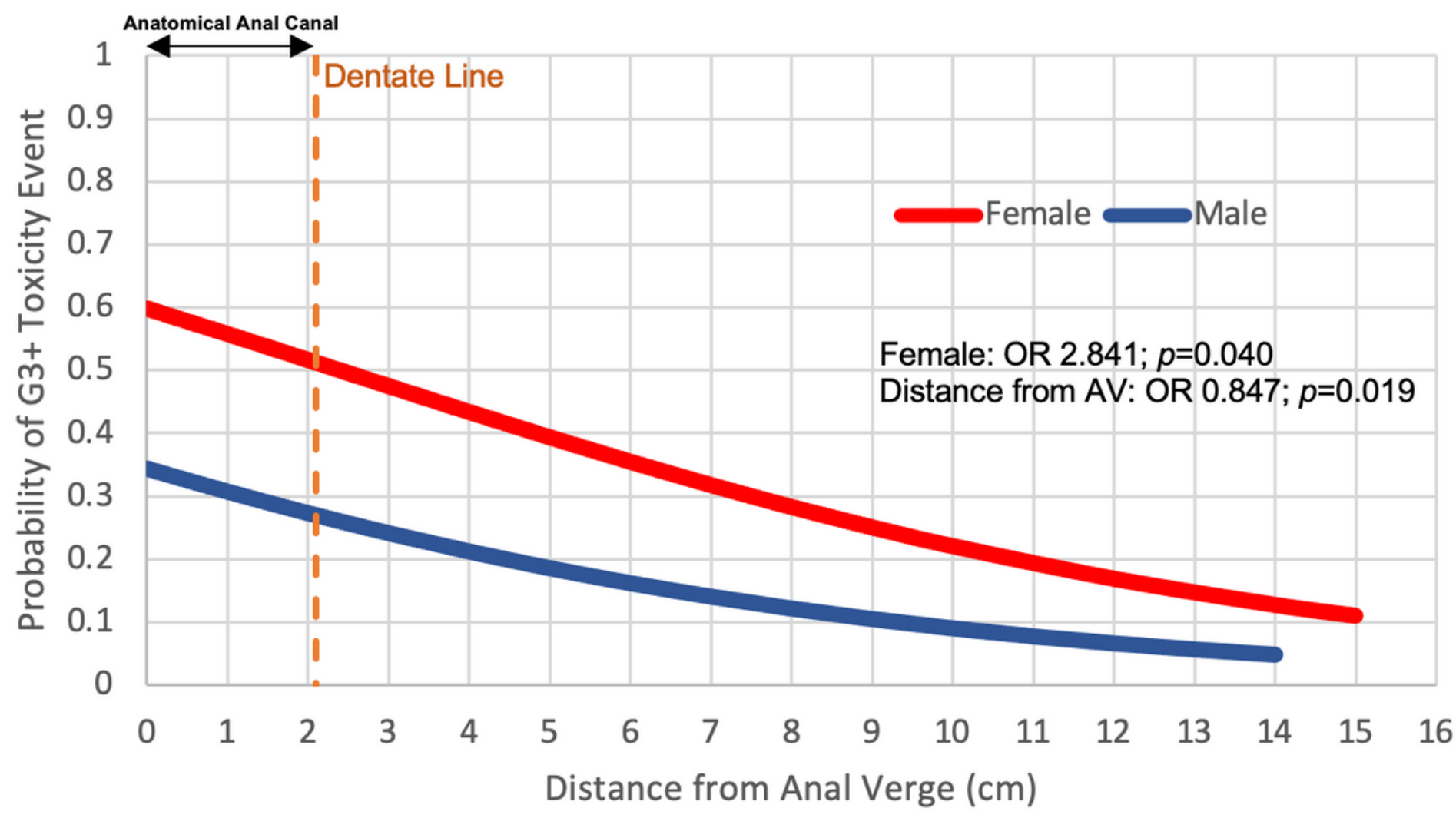

Figure 1

Factors affecting probability of grade $3+(\mathrm{G} 3+)$ toxicity event. Sex (male[ref]/female; Odds Ratio 2.841, p=0.040) and distance from the AV (continuous centimeters proximal from AV; Odds Ratio 0.847; $\mathrm{p}=0.019$ ). Dentate line: $2.1 \mathrm{~cm}$ proximal from AV (dashed orange line). 


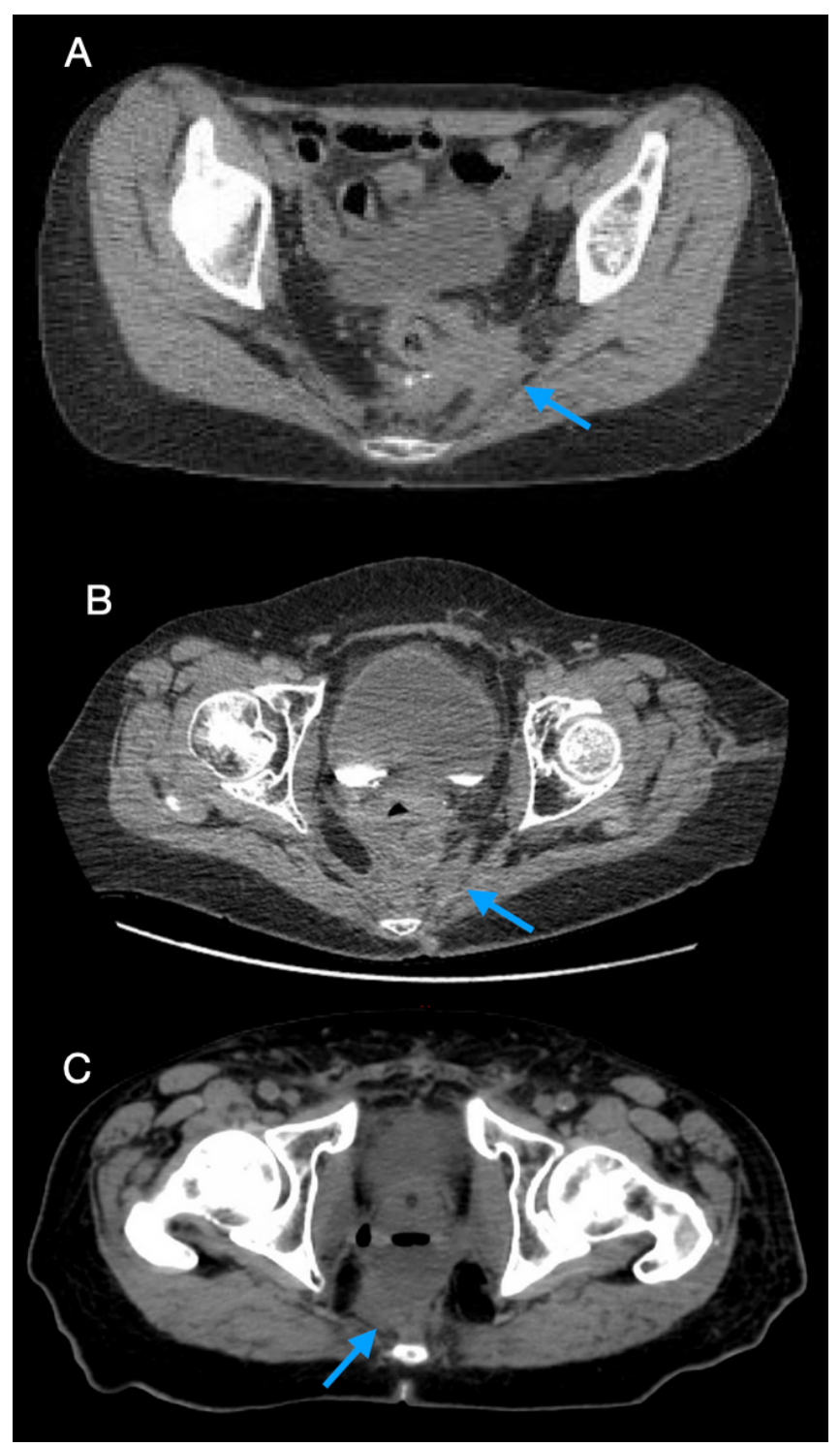

Figure 2

Axial computed tomography slices of the local failure events. Blue arrow: local recurrence. Case A: 52.3 years old female.

cT3cN0/LAR/ypT3ypN2a, negative margins. Isolated local recurrence after 77.7 months retreated with neoadjuvant chemotherapy and resection. Last status: alive with no evidence of disease (125.8 months). Case B: 53.7 years old female. cT3cN2b/APR/ypT3ypN2a, positive margin (distal). Local recurrence after 14.4 months with widespread distant failure before. Last status: cancer related death (15.4 months) Case C: 55.0 years old male. cT3cN1/LAR/ypT4bypN2b, positive margin (distal) and close margin (radial). Local recurrence after 11.1 months with widespread distant failure before. Treated with palliative radiotherapy $30 \mathrm{~Gy}$ (10 x 3.0Gy). Last status: cancer related death (19.4 months). 


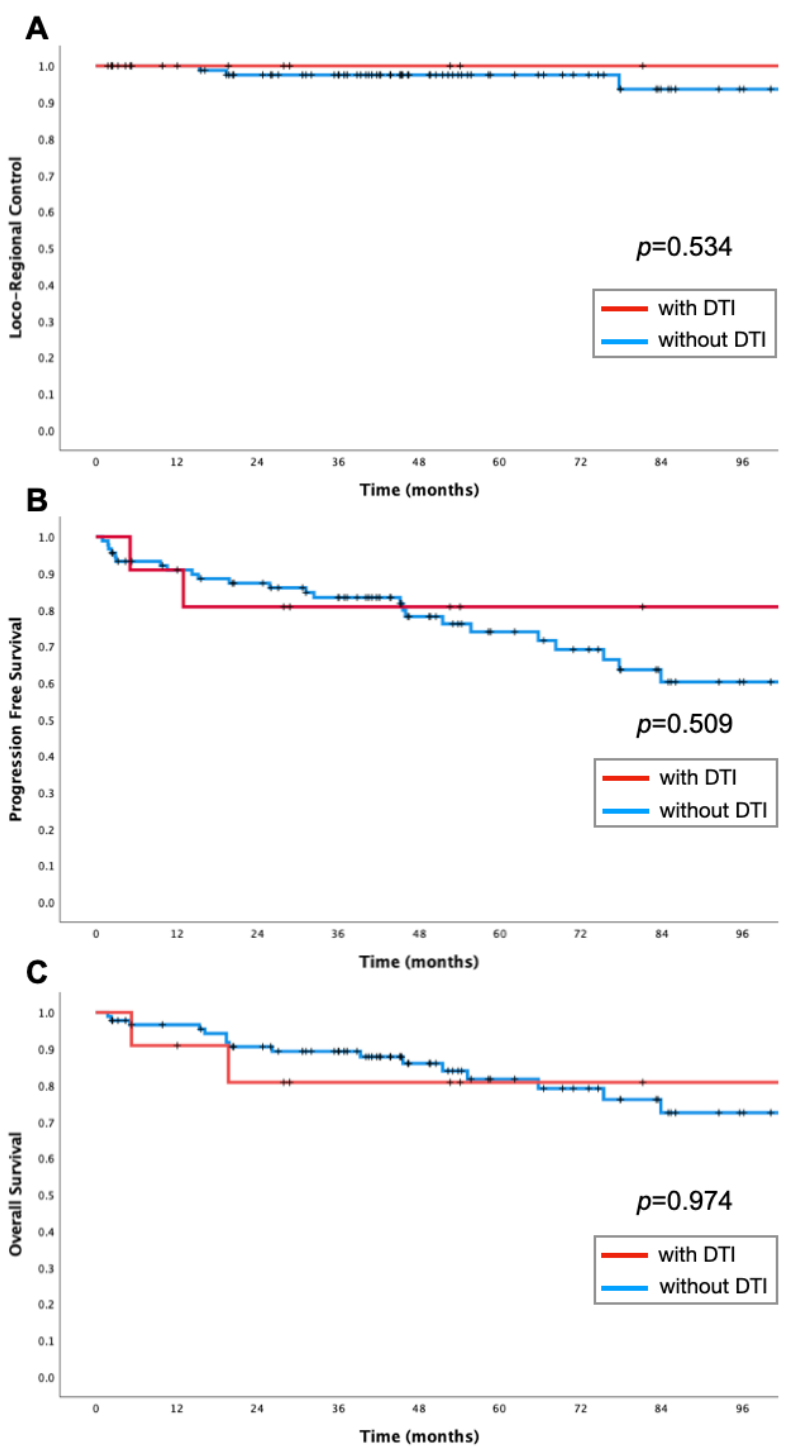

\section{Figure 3}

Kaplan-Meier representation of survival outcomes. A: Loco-regional control. B: Progression-free survival. C: Overall survival. Univariate (log-rank test). Blue line: cases without definitive treatment interruption (DTI). Red line: cases with DTI.

\section{Supplementary Files}

This is a list of supplementary files associated with this preprint. Click to download.

- SupplementaryAppendix.docx 